\title{
Original
}

\section{Microbial Contamination of Rubber Ducks Floating in Bathtubs of Bathing Facilities, and an Evaluation of Their Washing Methods}

\author{
AKIKO EDAGAWA ${ }^{1 *}$, NORIHIRO MATSUDA ${ }^{2}$, TORU OGURA ${ }^{3}$, KENICHI UEZONO $^{3}$, \\ SHINJI IZUMIYAMA ${ }^{4}$, AND AKIRA FUJII ${ }^{2}$ \\ ${ }^{1}$ Department of Environment Health, Osaka Institute of Public Health, 1-3-69 Nakamichi, \\ Higashinari-ku, Osaka-shi, Osaka 537-0025, Japan \\ ${ }^{2}$ Product Development \& Quality Control Department, Health Beauty Co., Ltd, \\ 2-5-8 kikusumi, Minami-ku, Nagoya-shi, Aichi 457-0012, Japan \\ ${ }^{3}$ Shyounankisai Yunoichi, 544 Karasawa, Fujisawa-shi, Kanagawa 251-0003, Japan \\ ${ }^{4}$ Department of Parasitology, National Institute of Infectious Diseases, \\ 1-23-1 Toyama, Shinjuku-ku, Tokyo 162-8640, Japan
}

Received 26 February, 2021/Accepted 21 June, 2021

\begin{abstract}
Microbiological contamination inside rubber ducks floating in the bathtub at a "duck bath" of a bathing facility was analyzed by examining bacterial and amoebic counts. The results of microbial tests, such as standard plate count, heterotrophic plate count and Legionella-LAMP (Loopmediated isothermal amplification), showed that microbial contamination increased in the rubber ducks over time. When the ducks were washed with sodium hypochlorite, those microbial contaminations were not detected; but when the ducks were washed with an electrolyzed water, the standard plate counts and the heterotrophic plate counts were detected in the amount of $10^{3}$ per duck in the wipe samples. Without proper washing of rubber ducks, bacteria and free-living amoeba can grow and colonize biofilms, and can thereby cause infection in humans. Also, microbial contamination inside ducks may reduce chlorination of the entire bathtub and cause bacterial infection such as Legionellosis from the bathtub water.
\end{abstract}

Key words : Rubber ducks / Microbial contamination / Legionella species / Free-living amoebae.

\section{INTRODUCTION}

Bathroom conditions are known to promote biofilm formation and growth due to moderately high temperatures and increased humidity. Plastic toys used in bathrooms become contaminated with bacteria (Neu et al., 2018). Many bathing facilities in Japan have attractions called "duck bath" in which many ducks float in the bathtub and adults and children alike enjoy them. The ducks are hollow inside and each has a small hole in the bottom, so water tends to accumulate inside. In a study of ducks collected from household bathrooms, biofilms formed inside ducks, and human pathogenic bacteria such as Pseudomonas aeruginosa and Listeria

*Corresponding author. TEL \& FAX: +81-6-6972-1321, E-mail : edagawa(a)iph.osaka.jp monocytogenes were detected in $80 \%$ of ducks while Legionella spp. was detected in 33\% of them (Neu et al., 2018). This suggests that rubber ducks used in duck bath in bathing facilities may cause microbial contamination of bath water and bacterial infections such as Legionella. One of the most important infectious diseases in bathtub environments is Legionnaires' disease in Japan, and the number of notifications is increasing year by year (National Institute of Infectious Diseases, Japan, 2020). Indeed, Legionella bacteria have been frequently detected in bathing facilities in Japan (Kanatani et al. 2017, Edagawa et al. 2015, Inoue et al 2015, Furuhata et al. 2004); and where hygiene control is inadequate, microbial contamination may be transmitted through ducks. In Japanese bathtubs of bathing facilities, chlorination is mandatory and Legionella is controlled. However, when chlorine is 
consumed by dirt inside ducks, the chlorine concentration in the bathtub water decreases, and Legionella and bacteria may proliferate and cause infection. To our knowledge, there are no reports of microbial contamination of toys, including rubber ducks, used in bathing facilities in Japan, though the bathing is very common among the Japanese.

Free-living amoebae are widely distributed in the aquatic environment and have been isolated from bathtub water, drinking water, and river water (Zbikowska et al. 2013, Shaheen et al. 2019, Saburi et al., 2017, Edagawa et al. 2009). Free-living amoebae serve as hosts for bacteria such as Legionella, Escherichia coli, Mycobacterium avium and P. aeruginosa, provide a breeding ground for a variety of bacteria (Greub et al., 2004), and are frequently detected in biofilms in the environment (Shaheen et al., 2019, Taravaud et al., 2018). Especially, in bathtub water, free-living amoebae are present at a high rate together with Legionella. Biofilm is a complex of many microorganisms, including bacteria and those amoebae, and microorganisms in biofilms are protected from chlorine disinfections.

A bathing facility in Kanagawa, Japan, found that their rubber ducks in duck baths were microbiologically contaminated similarly to those in the report (Neu et al., 2018). The test said that there were $10^{5}$ cfu per duck of standard plate counts (SPC) and heterotrophic plate counts (HPC), in addition to their Legionella-LAMP (Loop-mediated isothermal amplification) and freeliving amoebae that were positives. It was shown that biofilms had formed inside ducks, even though they were carefully and regularly washed with tap water and alcohol. In this study, we examined the growth of bacteria and free-living amoebae in new rubber ducks floated in bath water in a bathing facility to evaluate contamination. And we investigated a method for cleaning the inside of the ducks of microorganisms, in order to achieve the safety of the duck baths. The bathwater is circulating equipped with a sand filter and maintained at more than $0.2 \mathrm{mg} / \mathrm{L}$ of free chlorine in order to avoid Legionella growth.

\section{MATERIALS AND METHODS}

\section{Rubber duck and bath samples}

The rubber ducks used for this study (Fig. 1: Length $5.2 \times$ Width $5.3 \times$ Height $4.3 \mathrm{~cm}$, volume approximately $36 \mathrm{~cm}^{3}$, surface area approximately $70 \mathrm{~cm}^{2}$ ) were the identical to the ones regularly used in the bathing facility, but new in the sense that they had not been used in duck baths before. Squeezing and releasing the hollow plastic figure of a "rubber duck" will cause the exchange of air and/or water through the sprue hole left from its
A)

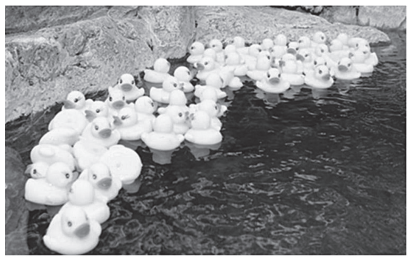

B)

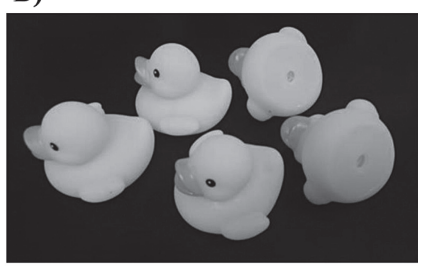

C)

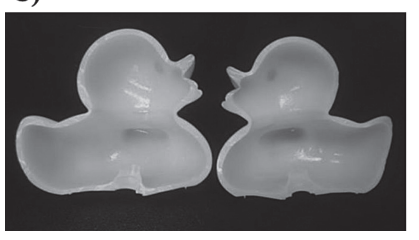

FIG. 1. Rubber ducks used in this study.

A) "Duck bath" with many rubber ducks floating

B) A rubber duck has a small hole in the bottom and a hollow inside.

C) Inside surface of rubber duck after use in the duck bath.

casting. And adults when bathing use this property to make bubbles of air under water, and squirt water into the air. The bath water was sampled by taking $500 \mathrm{~mL}$ of water in a sterilized bottle containing sodium thiosulfate at a final concentration of $0.1 \%$.

\section{Microbial contamination inside rubber ducks}

In order to investigate the biofilm formation inside the rubber ducks, the microbial growth during floating in the bathtub was measured. A model bathtub was installed in the bathing facility to create an environment similar to its bathtub water, and rubber ducks were floated. For the model bathtub, a plastic container of $513 \times 319 \times$ $156 \mathrm{~mm}$ was installed in the machine room of the bath facility, and the bathtub water was supplied continuously by a pump from a bathtub which was actually being used there, so the model bath was always filled and flowing with man-used water. The chlorine concentration in the facility's bathtub water was always maintained at $0.2 \mathrm{mg} / \mathrm{L}$ or more.

Some bath water typically remains in the ducks, which has entered through the sprue hole located on their bottom surface, after bathers play with them. In this study we intentionally filled the ducks with the bath water when floating ducks in order to accelerate the accumulation of pollution inside the ducks. Floating of the ducks filled with bathtub water was started (at 16:00), and after 2.5 hours, the water inside the ducks was squeezed in and out in the model bathtub (18:30), and the bathtub water was filled inside again. After 2.5 hours, the ducks were pulled out of the bathtub (21:00); and after sufficient water was drained, the ducks were placed with their bottom surface facing up, 
and dried. The ducks were floated daily in a similar manner to those used in the facility's duck bath, and after $1,3,5$, and 7 days, the duck's internal washing solution, internal wipe solution and the bathtub itself were tested for microorganisms.

\section{Microbiological test}

For the rubber duck internal washing sample, $10 \mathrm{~mL}$ of sterile distilled water was poured into each of 5 rubber ducks and mixed with a vortex mixer for 30 seconds. After being allowed to stand at room temperature for 1 hour, the mixture was mixed again with a vortex mixer for 30 seconds, and then the elute was collected into a test tube, mixed well, and used as a 50 $\mathrm{mL}$ wash sample of the ducks.

The five ducks after taking the internal washing samples above were cut in half in a straight line from the head to the tail, and the inside of each duck was wiped with a sterile cotton swab (Sato kasei kogyosyo Co., Ltd., Tochigi, Japan). The microbes were eluted into the tube with a $10 \mathrm{~mL}$ of phosphate buffered saline from the swabs by mixing, and then elutes were combined into a sample as a wipe sample of the ducks.

The samples were tested for microorganisms: SPC, HPC, free-living amoeba, and Legionella-LAMP. SPC and HPC were tested with $1 \mathrm{ml}$ of sample or diluted as necessary. SPC was determined on standard method agar (Nissui Pharmaceutical Co., Ltd., Tokyo, Japan) for 48 hours at $37^{\circ} \mathrm{C}$ (Japan water works association, 2011). HPC was determined by culturing on R2A agar (Nissui Pharmaceutical Co., Ltd., Tokyo, Japan) for 2 days at $37^{\circ} \mathrm{C}$ and 5 days at $25^{\circ} \mathrm{C}$ for a total of 7 days.

The isolation of free-living amoebae was detected by a culture method using filter filtration (Edagawa et al. 2009,). The samples of $20 \mathrm{~mL}$ each for the duck wash and the wipe solution and $100 \mathrm{~mL}$ for the bathtub water was filtrated through a cellulose nitrate filter (pore size, 1.2 um; diameter, $24 \mathrm{~mm}$, Millipore, Tokyo, Japan). Each filter was cut into eight pieces, inverted, and placed diagonally on two 1.5\% non-nutrient agar plates seeded with heat-killed Escherichia coli strain $\mathrm{DH} 1$ (i.e., four filters per a plate). With two plates per sample, one was then incubated at $30^{\circ} \mathrm{C}$ and the other at $42^{\circ} \mathrm{C}$ for up to 7 days until growth of amoeboid plaques became visible. The optimum temperatures for environmental free-living amoebae were $42^{\circ} \mathrm{C}$ for Naegleria and $30^{\circ} \mathrm{C}$ for Acanthamoeba and Vannella, and cultures were taken at the two temperatures for broad detection. During this incubation period, amoebic growth was examined daily by inverted phase microscopy. Amoeba plaques are formed by spreading from four filters per plate. Thus, the number of plaques formed per amoeba agar plate by this method was 0 for negative and $1+$ to $4+$ for positive, and this value was used to assess the degree of contamination.

Legionella-LAMP was performed according to the manufacturer's instructions by using a Loopamp Legionella Detection Kit E (Eiken Chemical Co., Ltd., Tokyo, Japan). The wipe and wash samples used approximately $30 \mathrm{~mL}$ of the remainder of the microbial test, and the bathtub water samples used five hundred milliliters. Each of these samples was concentrated by filtration through a $0.45-\mu m$-pore size mixed cellulose ester filter (Merck Millipore Co. Ltd., Darmstadt, Germany). The membrane was then immersed in $5 \mathrm{~mL}$ of sterile deionized water, and vortexed for $1 \mathrm{~min}$. From this suspension, $2 \mathrm{~mL}$ was used for Legionella-LAMP.

\section{Examination on washing method for inside of the rubber ducks}

The washing was carried out by two methods after their uses: M1) drying and internal washing, and M2) immersion in a washing solution. M1: the rubber duck was dried overnight at $40^{\circ} \mathrm{C}$ after adequate drainage of internal moisture. Thereafter, the washing liquid was taken in and out three times to wash the inside. M2: the washing liquid was taken in and out three times, and the rubber ducks were left to stand overnight with the washing liquid filled inside. The washing solutions were then sufficiently discharged.

The three kinds of washing solution were: S1) tap water, S2) slightly acidic electrolyzed water, and S3) sodium hypochlorite which was adjusted to a concentration of $60 \mathrm{mg} / \mathrm{L}$ by diluting a commercial household product. The electrolyzed water (Purester, Morinaga milk industry Co., Ltd., Tokyo, Japan) in this study is used daily in the kitchen of the bathing facility. The water is designated as a food additivein addition to its washing purpose, and has been recognized for the effect of sterilizing bacteria (Suzuki et al. 2005, Nakamura et al. 2010). The concentration of effective chlorine and the $\mathrm{pH}$ value would be 20 to $60 \mathrm{ppm}$ and 2.2 to 2.7 respectively, according to the instruction manual.

\section{RESULTS AND DISCUSSION}

\section{Microbial growth inside rubber ducks}

Table 1 and Fig. 2 showed the time course of microbial contamination in the rubber ducks floating in the model bath. The numbers of SPC and HPC increased day by day. After 5 days, the washing and wipe samples were $10^{7} \mathrm{CFU}$ and $10^{6} \mathrm{CFU}$ per duck, respectively. In a blank test of the washed samples using completely unused ducks, the number of SPC was $1 \times 10^{2}$ CFU per duck, the number of HPC was $2 \times 10^{1} \mathrm{CFU}$ per duck, and the numbers of these bacteria in the wipe samples were both less than 10 CFU per duck. The 
TABLE 1. Results of Legionella LAMP and free living amoebae detection inside rubber ducks

\begin{tabular}{|c|c|c|c|c|c|c|}
\hline \multirow{3}{*}{ day } & \multicolumn{3}{|c|}{ Free living amoebae $^{1)}$} & \multicolumn{3}{|c|}{ Legionella-LAMP } \\
\hline & \multicolumn{2}{|c|}{ Rubber duck } & \multirow{2}{*}{$\frac{\text { Bathtub water }}{30^{\circ} \mathrm{C} / 42^{\circ} \mathrm{C}}$} & \multicolumn{2}{|c|}{ Rubber duck } & \multirow[b]{2}{*}{ Bathtub water } \\
\hline & $\begin{array}{c}\text { Wash }^{2)} \\
30^{\circ} \mathrm{C} / 42^{\circ} \mathrm{C}\end{array}$ & $\begin{array}{c}\text { Wipe }^{3)} \\
30^{\circ} \mathrm{C} / 42^{\circ} \mathrm{C}\end{array}$ & & Wash $^{2)}$ & Wipe $^{3)}$ & \\
\hline 1 & $2+1-$ & $-1-$ & $4+/ 4+$ & + & - & + \\
\hline 3 & $4+/ 4+$ & $3+1-$ & $1+/ 4+$ & - & - & - \\
\hline 5 & $4+/ 4+$ & $4+/ 4+$ & $4+/ 4+$ & - & - & + \\
\hline 7 & $4+/ 4+$ & $4+/ 4+$ & $4+/ 4+$ & + & - & + \\
\hline
\end{tabular}

1) The number of plaques formed per amoeba agar plate was 0 for negative and $1+$ to $4+$ for positive, and this value was used to indicate the degree of contamination.

${ }^{2)}$ Washing solution inside a rubber duck, ${ }^{3)}$ Wiping the inner surface of a rubber duck

A)

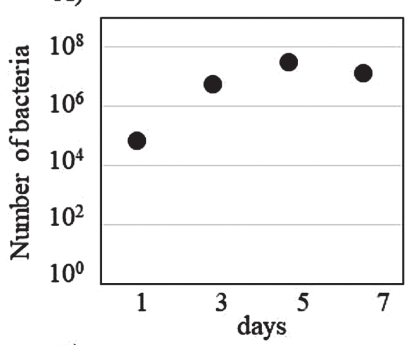

C)

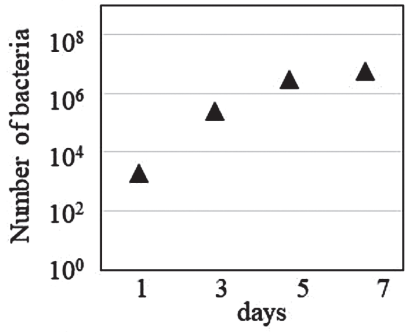

E)

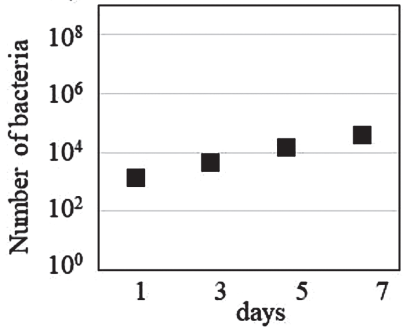

B)

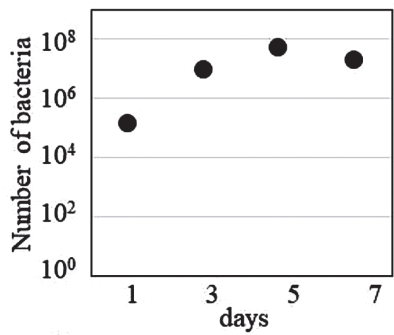

D)

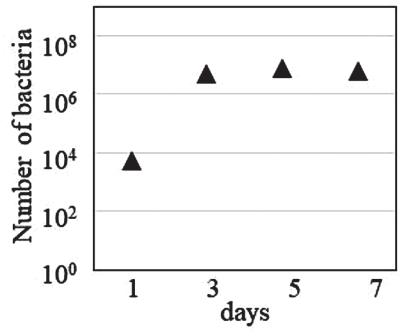

F)

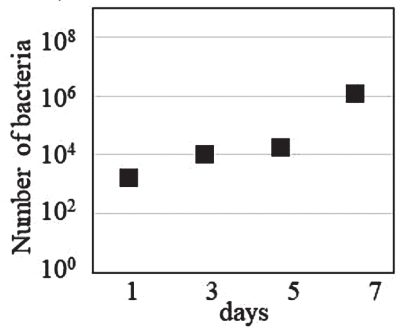

FIG. 2. Growth of microorganisms inside rubber ducks and bathtub waters
A) Washing solution inside a rubber duck (CFU/duck): Standard plate count bacteria
B) Washing solution inside a rubber duck (CFU/duck): Heterotrophic bacteria
C) Wiping the inner surface of a rubber duck (CFU/duck): Standard plate count bacteria
D) Wiping the inner surface of a rubber duck (CFU/duck): Heterotrophic bacteria
E) Bath water $(\mathrm{CFU} / \mathrm{mL})$ : Standard plate count bacteria
F) Bath water $(\mathrm{CFU} / \mathrm{mL})$ : Heterotrophic bacteria

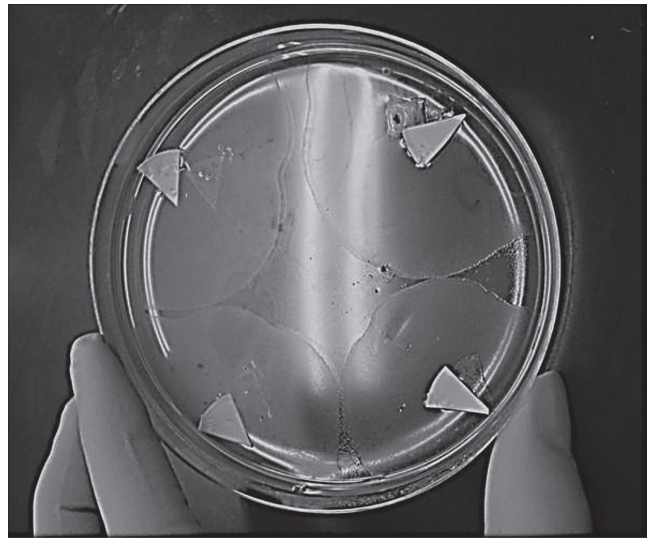

FIG. 3. Amoebic plaques formed on the free-living amebae test agar plates $(4+)$

microbial growth of SPC and HPC in ducks was 2 to 3 Log higher than that of unused ducks. Bacterial counts increased 1 to 3 Log after 3 days and 2 to 3 Log after 5 days compared with after 1 day alone. Among SPC and HPC of the wash and wipe samples, the number of SPC was highest in the wipe sample after 7 days, but in other items, it was higher after 5 days than after 7 days. Therefore, these results suggest that bacteria grow inside ducks 1 to 3 days after the start of floating in the bathtub, and that biofilm colonizes the ducks 5 days later.

The free-living amoeba test, which determines the degree of microbial contamination of the bathtub samples, was well performed at both of these temperatures. The amoebae lived in high concentrations of $4+$ from the first day (Fig. 3), although the concentration of chlorine was secured around $0.2 \mathrm{mg} / \mathrm{L}$ in the bathtub water. Soon after the starting of the model bath, the wipe samples showed amoeba positives. Legionella-LAMP was positive on day 1 , negative on days 3 and 5 , and positive again on day 7 . Since all of the wipe samples were negative for Legionella-LAMP, Legionella is likely 
TABLE 2. Results of microorganism detection inside rubber ducks for each washing method

\begin{tabular}{|c|c|c|c|c|c|c|c|c|c|}
\hline \multirow{2}{*}{ Washing method } & \multirow{2}{*}{ Washing solution } & \multicolumn{2}{|c|}{$\begin{array}{l}\text { Standard plate } \\
\text { (ount }{ }^{1)}\end{array}$} & \multicolumn{2}{|c|}{$\begin{array}{c}\text { Heterotrophic plate } \\
\text { count } t^{1)}\end{array}$} & \multicolumn{2}{|c|}{$\begin{array}{l}\text { Free living } \\
\text { amoebae }^{2)}\end{array}$} & \multicolumn{2}{|c|}{$\begin{array}{l}\text { Legionella- } \\
\text { LAMP }\end{array}$} \\
\hline & & wash & wipe & wash & wipe & $\begin{array}{l}\text { Wash } \\
30^{\circ} \mathrm{C} / 42^{\circ} \mathrm{C}\end{array}$ & $\begin{array}{c}\text { Wipe } \\
30^{\circ} \mathrm{C} / 42^{\circ} \mathrm{C}\end{array}$ & wash & wipe \\
\hline \multirow{3}{*}{$\begin{array}{l}\text { M1: drying overnight } \\
\text { and washing }\end{array}$} & S1: tap water & $6.1 \times 10^{3}$ & $2.5 \times 10^{3}$ & $1.7 \times 10^{4}$ & $5.7 \times 10^{3}$ & $2+/-$ & $-1-$ & - & + \\
\hline & $\begin{array}{l}\text { S2: Slightly acidic electro- } \\
\text { lyzed water }\end{array}$ & 0 & $2.1 \times 10^{3}$ & 0 & $2.6 \times 10^{3}$ & $-1-$ & $-1-$ & - & - \\
\hline & $\begin{array}{l}\text { S3: Sodium hypochlorite } \\
\text { aqueous solution }{ }^{3)}\end{array}$ & 0 & 0 & 0 & 0 & $-1-$ & $-1-$ & - & - \\
\hline \multirow{3}{*}{$\begin{array}{l}\text { M2: Immerse in a } \\
\text { washing solution } \\
\text { overnight }\end{array}$} & S1: tap water & $1.5 \times 10^{5}$ & $2.3 \times 10^{4}$ & $2.3 \times 10^{5}$ & $2.6 \times 10^{4}$ & $4+/-$ & $-1-$ & + & - \\
\hline & $\begin{array}{l}\text { S2: Slightly acidic electro- } \\
\text { lyzed water }\end{array}$ & 0 & 0 & 0 & 0 & $-1-$ & $-1-$ & - & - \\
\hline & $\begin{array}{l}\text { S3: Sodium hypochlorite } \\
\text { aqueous solution }{ }^{3)}\end{array}$ & 0 & 0 & 0 & 0 & $-1-$ & $-1-$ & - & - \\
\hline
\end{tabular}

${ }^{1)} \mathrm{CFU}$ per duck, ${ }^{2)}$ The number of plaques formed per amoeba agar plate was 0 for negative and $1+$ to $4+$ for positive, and this value was used to indicate the degree of contamination. ${ }^{3)} 60 \mathrm{mg} / \mathrm{L}$

not colonized. Since bathwater Legionella-LAMP was positive at 1,5 , and 7 days, it is likely that LegionellaLAMP was positive due to the influence of the Legionella gene present in bathwater. It is unlikely that Legionella colonizes and proliferates internally in this study, but it may be easy for those Legionella to colonize inside the ducks on one of those days. Those rubber ducks should not be used continuously and should be washed both inside and outside after each day of use before the establishment of free-living amoebae.

\section{Microbial test results by washing method}

Table 2 shows the results of microbial tests according to the washing method. The washing with sodium hypochlorite was the most effective regardless of the method. An electrolyzed water was not sufficiently effective when washed after drying overnight, but was sufficiently effective when immersing overnight. The tap water was not effective at all. These results indicate that those ducks require at least the use of sodium hypochlorite and/or the immersing overnight in electrolyzed water.

The immersing overnight in electrolyzed water would be one practical solution as a countermeasure against microbial contamination, since several hundred ducks are used at a time in the public duck bath, and the washing work is very heavy. The electrolyzed water should be delivered continuously to the ducks in a large bucket for this purpose so as to keep the concentration of the disinfectant high enough. Unfortunately, rubber ducks cannot be sterilized by heating, because the plastic used in them quickly deteriorates.

In this study, we found that the interior of rubber ducks used in bathing facilities are contaminated with microorganisms if they are not properly washed. Because our ducks were washed regularly with ethanol, our duck (Fig. 1C) seems to be cleaner than the one described in other reports, and we can see a shocking picture showing the dirty inside of a bath toy in Figure 1a (Neu et al., 2018). There was some possibility of ethanol shortage if some water remains inside the ducks, for then dilution occurs and the effectiveness of the disinfection will decrease. Indeed, even under the conditions used in this study, the test detected some contamination with Legionella and amoebae in the ducks, and we had to improve the way of washing the ducks immediately. As a result of examining the washing method, sufficient effect was obtained by washing with sodium hypochlorite or immersing in electrolyzed water overnight. Without proper washing of rubber ducks, bacteria and free-living amoeba can grow and colonize biofilms, and can thereby cause infection in humans. Also, microbial contamination inside ducks may reduce chlorination of the entire bathtub water and cause bacterial infection such as Legionella from the bathtub water.

\section{ACKNOWLEDGMENTS}

This study was supported partially by a Health and Labor Sciences Research Grant (19LA1006) from the Ministry of Health, Labor and Welfare, Japan.

\section{REFERENCES}

Edagawa, A., Kimura, A., Kawabuchi-Kurata, T., Kusuhara, Y. and Karanis, P. (2009) Isolation and genotyping of potentially pathogenic Acanthamoeba and Naegleria species 
from tap-water sources in Osaka. Japan. Parasitol Res., 105, 1109-17.

Edagawa, A., Kimura, A., Kawabuchi-Kurata, T., Adachi, S., Furuhata, K. and Miyamoto, H. (2015) Investigation of Legionella Contamination in Bath Water Samples by Culture, Amoebic Co-Culture, and Real-Time Quantitative PCR Methods. Int J Environ Res Public Health., 19, 13118-30.

Furuhata, K., Hara, M., Yoshida, S., and Fukuyama, M. (2004) Distribution of Legionella spp. in hot spring baths in Japan. (in Japanese), Kansenshogaku Zasshi, 78, 710716.

Greub, G., and Raoult, D. (2004) Microorganisms resistant to free-living amoebae. Clin. Microbiol. Rev., 17, 413-33.

Inoue, H., Takama, T., Yoshizaki, M., and Agata, K. (2015) Detection of Legionella species in environmental water by the quantitative PCR method in combination with ethidium monoazide treatment. Biocontro/ Sci., 20, 71-4

Japan water works association (2011) Standard Methods for the Examination of Water (in Japanese).

Kanatani, J., Isobe, J., Norimoto, S., Kimata, K., Mitsui, C., Amemura-Maekawa, J., Kura, F., Sata, T. and Watahiki, M. (2017) Prevalence of Legionella species isolated from shower water in public bath facilities in Toyama Prefecture, Japan. J Infect Chemother., 23, 265-70.

Nakamura, T., Oda, H., and Sato, S. (2010) Effects of slightly acidic electrolyzed water on oral pathogens and human Gingival Fibroblasts. (in Japanese), JSCD., 53, 570-578.

National Institute of Infectious Diseases, Japan (2020)
Infectious Diseases Weekly Report (IDWR)

https://www.niid.go.jp/niid/en/

Neu, L., Bänziger, C., Proctor, CR., Zhang, Y., Liu, WT., and Hammes, F. (2018) Ugly ducklings-the dark side of plastic materials in contact with potable water. NPJ Biofilms Microbiomes., 4, 7.

Saburi, E., Rajaii, T., Behdari, A., Kohansal, MH., and Vazini, H. (2017) Free-living amoebae in the water resources of Iran: a systematic review. J Parasit Dis., 41, 919-28.

Shaheen, M., Scott, C., and Ashbolt, NJ. (2019) Long-term persistence of infectious Legionella with free-living amoebae in drinking water biofilms. Int J Hyg Environ Health., 222 678-86.

Suzuki, K., Nakamura, T., Kokubo, S., and Tomita, M. (2005) The physical properties of slightly acidic electrolyzed water prepared with hydrochloric acid as a raw materia. (in Japanese) Bokin Bobai, 33, 55-62.

Taravaud, A., Ali, M., Lafosse, B., Nicolas, V., Féliers, C., Thibert, S., Lévi, Y., Loiseau, PM., and Pomel, S. (2018) Enrichment of free-living amoebae in biofilms developed at upper water levels in drinking water storage towers: An inter- and intra-seasonal study. Sci Total Environ., 15, 15766.

Zbikowska, E., Walczak, M., and Krawiec, A. (2013) Distribution of Legionella pneumophila bacteria and Naegleria and Hartmannella amoebae in thermal saline baths used in balneotherapy. Parasitol Res., 112, 77-83. 